\title{
Co-Designing Smart Home Technology with People with Dementia or Parkinson's Disease
}

\author{
Aikaterini Bourazeri \\ Centre for HCI Design \\ City, University of London \\ London, UK \\ Katerina.Bourazeri@city.ac.uk
}

\author{
Simone Stumpf \\ Centre for HCI Design \\ City, University of London \\ London, UK \\ Simone.Stumpf.1@city.ac.uk
}

\begin{abstract}
Involving users is crucial to designing technology successfully, especially for vulnerable users in health and social care, yet detailed descriptions and critical reflections on the co-design process, techniques and methods are rare. This paper introduces the PERCEPT (PERrsona-CEntred Participatory Technology) approach for the co-design process and we analyse and discuss the lessons learned for each step in this process. We applied PERCEPT in a project to develop a smart home toolset that will allow a person living with early stage dementia or Parkinson's to plan, monitor and self-manage his or her life and well-being more effectively. We present a set of personas which were cocreated with people and applied throughout the project in the co-design process. The approach presented in this paper will enable researchers and designers to better engage with target user groups in co-design and point to considerations to be made at each step for vulnerable users.
\end{abstract}

\section{Author Keywords}

Co-design; personas; dementia; Parkinson's disease.

\section{ACM Classification Keywords}

H.5.2 User Interfaces: Theory and Methods; H.5.m. Information interfaces and presentation (e.g., $\mathrm{HCI}$ ): Miscellaneous.

\section{INTRODUCTION}

There are many ways to involve users in the design of technology, and a myriad of techniques and methods have been suggested to create empathy with users and their experience in order to design technology [44]. Ways to involve users range from "designing-for" approaches in which users' needs are captured and analysed by researchers, and then translated and implemented into solutions by designers, to "designing-with" approaches, characterized by

Permission to make digital or hard copies of all or part of this work for personal or classroom use is granted without fee provided that copies are not made or distributed for profit or commercial advantage and that copies bear this notice and the full citation on the first page. Copyrights for components of this work owned by others than the author(s) must be honored. Abstracting with credit is permitted. To copy otherwise, or republish, to post on servers or to redistribute to lists, requires prior specific permission and/or a fee. Request permissions from Permissions@acm.org.

NordiCHI'18, September 29-October 3, 2018, Oslo, Norway

(C) 2018 Copyright is held by the owner/author(s). Publication rights licensed to $\mathrm{ACM}$.

ACM ISBN 978-1-4503-6437-9/18/09 ..\$15.00

https://doi.org/10.1145/3240167.3240197 a deep and direct involvement of a targeted user group in all stages of the design process. Two well-known methods to design with users are participatory design [3], which has its roots in Scandinavia to include workers in the design of taskbased solutions, and co-design, which is a more recent, broader method that includes co-creation at the early "fuzzy" stages [32]. It has been argued that co-design is especially important for technology in health and social care to ensure its successful adoption and greater patient empowerment [16], such as technology targeting people with dementia and Parkinson's disease. Yet how to co-design successfully is rarely detailed in many publications.

While there is some previous work that reports on involving people with dementia or Parkinson's in designing technology $[20,37,38]$, the majority of techniques employed use interviews and design probes to stimulate discussion. Other projects involving older users or people with Parkinson's have employed the OASIS method, which uses preconstructed videos as prompts for workshop participants to explore and prototype technology [19,24]. In addition, there is very limited previous research about co-creating personas with end-users [8,9], especially in health and social care technology design. Publications that share with researchers and designers what techniques and methods were used in codesign, especially with vulnerable groups [36,35], and a critical refection on their applicability and effectiveness, are unfortunately very rare.

In this paper, we introduce the PERCEPT (PERrsonaCEntred Participatory Technology) approach and apply it in a case study. We describe the steps and techniques used in the PERCEPT approach in detail, including how to co-create personas, and how to use them to give structure to the codesign process. We analyse the benefits and limitations of our approach in the form of lessons learned for each step, in the hope of providing useful pointers for researchers and designers, especially those who are new to co-design or working with vulnerable groups. Our aim was to develop successful ways of engaging with our target users and to integrate them as equal partners in the design of technology. Our research questions were:

RQ1: How can we engage people with early stage dementia or Parkinson's, and their informal carers as equal research and design partners in co-design? 
RQ2: Is it feasible to co-create personas with people with dementia or Parkinson's?

RQ3: How can these personas be leveraged in the co-design process?

Our work is set in the context of developing EnableSelfCare, a new intelligent toolset of software, hardware and sensors that will allow a person living in their own home, together with their informal carers, to create, change and monitor a quality of life plan (the life plan). They will interact with the toolset through a user interface to describe and change the goals and activities that are to be achieved in the life plan. This information will then be used to configure low-cost sensors to collect data about these activities and a computational model will use the sensor data to infer whether desired activities and goals in the life plan are being achieved. This paper reports on the co-design activities we undertook as part of developing the user interface to link a computational quality of life model and sensor technology. This toolset is targeted initially at people living with early stage dementia or Parkinson's disease, although we envision that it could be adapted in future to other long-term, chronic diseases. We present a set of personas that were co-created by people with dementia and their informal carers and with people with Parkinson's to shape EnableSelfCare.

This paper will share the techniques we used as part of the PERCEPT approach, and lessons we learned from them; our purpose is not to describe and evaluate the technology we eventually developed. Our contributions are to offer suggestions to researchers and designers for integrating a toolkit of techniques into the co-design process, potentially adopt the PERCEPT approach themselves, and make considerations for co-designing with people with early stage dementia or Parkinson's and other vulnerable groups.

\section{RELATED WORK}

\section{Co-designing Health and Social Care Technology}

There are a number of ways to involve users in designing technology. In this paper, we will focus on co-design and participatory design approaches.

There have been increasing calls to actively involve users through a process of co-design [32,7], especially in a health and social care setting $[16,43]$. Co-design means not only asking users what they want, but treating them on a par in cocreation as researchers [32], and having equal creative input as designers in developing the solutions [26]. It is easy to pay lip service to co-designing when in fact users are only consulted; a fine example of involving users through codesign is the KITE project [31] which developed an armband and a notepad technology to maintain independence by deeply involving people with dementia and their informal carers.

There are many techniques which could be adapted to codesigning health and social care technology to create empathy and create effective solutions [44]. Usually, designers, researchers and users come together in co-design workshops, using artefacts such as photographs, drawings, and prototypes to explore and develop solutions [7]. Using probes $[15,21]$ is another technique that allows users to provide input into the co-design process, which has been used to design technology with people with dementia or Parkinson's [20,37,38]. The KITE project [31] developed technology for people with dementia through a staged design process comprising focus groups, workshops and meetings with people with dementia and their carers to scope the technology, and design and develop prototypes. The OASIS method $[19,24]$ for co-designing consists of four stages: stakeholder identification and recruitment, video creation to be used as prompts during the ensuing exploratory meetings that gather information about initial requirements and envisioning the system, and low-fidelity prototyping sessions that are structured around functionality, aesthetics, and experience. While recently different co-design approaches are starting to be shared among researchers and practitioners, a detailed description and critical reflection on techniques and methods employed as part of co-designing are rare.

There have been previous efforts to use personas in codesigning health and social care solutions $[34,39,42]$ as fictitious representations of user groups - "hypothetical archetypes" of target users - and their goals, needs and preferences [1,10]. Personas can be pre-created by researchers and then introduced into the co-design process [42]; occasions described in the literature when personas are co-created with users themselves are very infrequent and usually only include pre-defined facets that flesh out the persona's background such as likes, dislikes, hobbies, interests, behaviour patterns, and friendships [39]. There is a large body of research of how researchers can construct personas through gathering qualitative and/or quantitative data $[10,1,13,23,29]$ and how they can be used in the design process $[14,22,27,6]$. However, there is very limited work on co-creating personas with end-users $[8,9]$, and how these personas can be employed in co-designing health and social care technologies.

\section{Self-care Technology for People with Dementia or Parkinson's Disease}

We provide a very brief overview of dementia and Parkinson's; both the Alzheimer's Society (https://www.alzheimers.org.uk/) and Parkinson's UK (https://www.parkinsons.org.uk/) provide an excellent description for further details. Dementia is a group of related symptoms associated with an ongoing decline of brain functioning, including memory loss, problems concentrating, difficulties carrying out familiar tasks, being confused about time and place, mood changes. There are many different causes of dementia. Parkinson's disease is a neurodegenerative disorder which leads to progressive deterioration of motor function, including tremor, stiffness, slowness, impaired balance, and later on a shuffling gait. Every person living with dementia or Parkinson's will have 
specific symptoms, depending on their individual rate of progression, etc. and we targeted people with cognitive impairment and early stages of dementia and Parkinson's.

Self-care and self-management technology in health and social care can employ a variety of devices and applications, e.g. wearables, mobile apps, and web applications $[2,41,17]$. Some of these devices and applications help to overcome or limit the impairment experienced by people with dementia or Parkinson's, such as MindMate (http://www.mindmateapp.com/) which offers reminders or Dragon Naturally Speaking (http://www.nuance.co.uk/dragon/index.htm), which is Assistive Technology that replaces keyboard and mouse input with voice input. Other applications provide simple forms of therapy, such as CleverMind (http://myclevermind.com/), store a care plan, provide information on conditions, support people to track and monitor their condition, or provide recommendations for maintaining or improving their health [e.g. 28,18,4,25]. Smart home technology is recently gaining in popularity to monitor people's health, however most of its use is in specially constructed homes that embed sophisticated sensors for use by formal carers or health and social care professionals [5,11]. Our case study aims to explore technology that retro-fits low-cost sensors in users' own homes, not to measure their health information but instead to support them maintaining or improving their quality of life.

\section{THE PROJECT AND CO-DESIGN SET-UP}

We apply our new co-design approach in a case study that will develop a toolset to allow a person together with their informal carers to self-manage their everyday life in order to increase or maintain its quality. In this toolset, information from smart home sensors along with self-reported data will be fed into a computational model to monitor how well the individual is managing their activities and goals, and then to suggest changes or improvements the person can make based on their performance. This toolset is targeted initially at

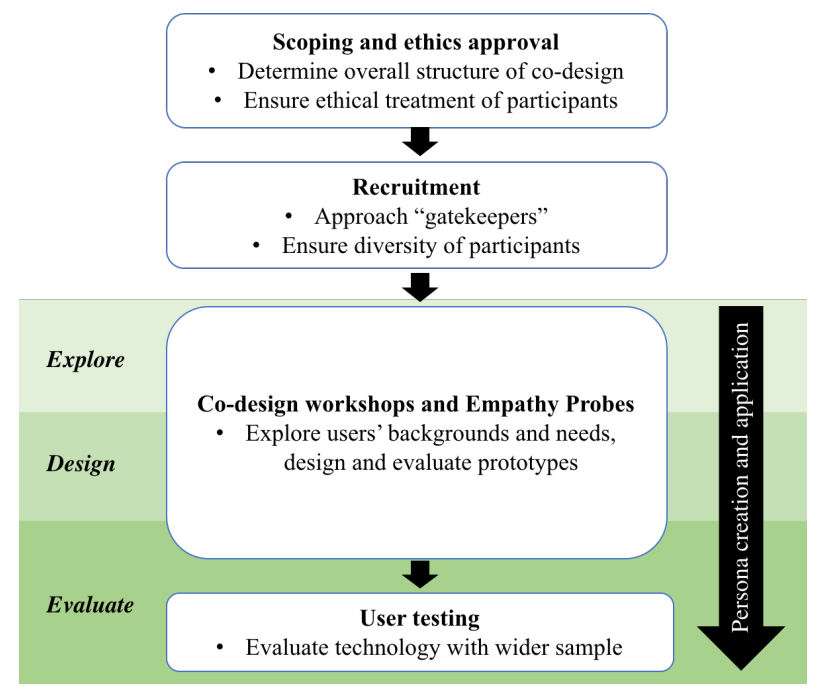

Figure 1. An overview of the PERCEPT approach. people living with early stages of dementia or Parkinson's Disease. The co-design process in which the PERCEPT approach was applied focused on the design of the user interface for creating and managing the life plan, but also provided input to the design of the wider system, e.g. the computational model and the sensors.

Over the course of twelve months, we undertook the following steps (Figure 1): scoping and ethics approval, recruitment, and exploration, design and evaluation steps including a series of co-design workshops, the use of empathy probes, and user testing. At the heart of the PERCEPT approach is co-creating and applying personas with target users during the exploration, design and evaluation steps. We now explain each of these steps in more detail, including how personas were co-created and applied by target users in our case study, and then we present the personas that were created and used throughout this codesign process. We reflect on and analyse our process by discussing the lessons learned for each of these steps that might be useful to other researchers, designers and practitioners.

\section{SCOPING AND ETHICS APPROVAL}

As our target group includes vulnerable adults with dementia who might have reduced capacity to consent, ethical approval was obtained through our university's research ethics committee and we sought advice from a nurse who specialises in the care of older people and people with dementia. The process of obtaining informed consent took into consideration the cognitive problems that participants with dementia might have and their ability to provide informed consent, following British law (Mental Capacity Act 2005) and guidelines developed by the British Psychological Society (BPS). The information and informed consent form provided to all participants was printed in large font in simple and easy-to-understand language, following the Dementia Engagement and Empowerment Project (DEEP) guidelines. All researchers with direct contact with vulnerable people underwent specialist training, and completed background checks. During the co-design workshops, we needed to establish if participants had lost mental capacity and thus the ability to consent. In addition, the workshop structure and venue were designed to accommodate mobility issues or reduced attention span of participants. Therefore, frequent breaks were scheduled through the workshop sessions and a separate quiet room was available for participants to take time out if required. Researchers and a specialist nurse were alert to signs that might have indicated that participants wished to stop.

As part of the ethical approval, we had to submit a detailed study plan, participant information, consent forms and recruitment materials. While we were able to be flexible about how to carry out the activities in detail, the aims and structure of the co-design steps could not be adjusted without obtaining further ethical approval. 


\section{Lessons Learned}

In total, we spend three months on obtaining initial ethics approval, which equates to $25 \%$ of the time allocated to codesigning within the project. We did not view this as time wasted; in fact, it surfaced important preconceptions about the abilities of our participants, and how to protect them adequately, supported by a wealth of advice from project team colleagues, and relevant guidelines, training materials, and laws and regulations. Computer scientists, HCI researchers and designers need to fully immerse themselves in the ethical considerations surrounding vulnerable participants as well as all individuals involved in co-design.

To participate in the co-design of EnableSelfCare, we required participants to be able to give informed consent, which meant we restricted ourselves to involving people with in the early stages of dementia. It is interesting to consider how to involve people who might not be able to give informed consent as co-designers in these types of projects, such people with later stages of dementia. Informant design [33] and cooperative inquiry [12], which were developed to design with children, a different vulnerable group, are possible approaches to consider that offer varying degrees of equality with researchers and designers.

We also decided to add user testing in month 12 as another step in our co-design; we sought an amendment to our ethical approval in month 10 . While it is not impossible to change the structure of the co-design process, it indicates that when working with potentially vulnerable people, researchers are often committed very early to following a particular procedure, and thus are less able to experiment with different techniques and methods.

\section{RECRUITMENT}

We targeted people with Parkinson's disease or early stage dementia who were living in their own homes to participate in our research. Over a period of two months, we recruited participants through advertising the study in email newsletters of relevant support organisations, such as Alzheimer's Society and Parkinson's UK, and visiting local dementia support groups in person.

Initially, we recruited six people - Adam ${ }^{1}$, Brian, Paul, Prabhu, Sarah, Gareth - with Parkinson's disease. After the first workshop, unfortunately Gareth had to withdraw from participating in further workshops. We also recruited three people - George, Arthur, Helen - with dementia, who each had an informal carer - George's wife June, Arthur's friend Andrew, and Helen's daughter Deirdre. After the first workshop, Helen's health deteriorated and she could no longer attend. Arthur lost interest in participating in this project, and also withdrew. Through a new round of recruiting via dementia support groups, we involved Colin and his live-in helper Paula. We repeated the first workshop

\footnotetext{
${ }^{1}$ All names changed to protect the participants' identity.
}

that they had missed, so they could join the second workshop with George and his wife.

We decided to have separate groups comprising people with Parkinson's and a group of people with dementia and their informal carers, mainly to keep workshops to a reasonable size and because needs differ substantially between these two conditions. The Parkinson's group consisted of 5 males and 1 female with an average age of 65 . They had a range of different symptoms, ranging from very slight tremors to extensive tremors and speech problems. Sarah and Paul were still working while Adam, Brian and Prabhu were retired. All were fiercely independent and insisted that they did not want their informal carers to come along. In the final dementia group, there were two males with an average age of 75 , with two female carers with an average age of 60 . Both Colin and George were retired, but tried to stay active and social through services offered locally or through the Alzheimer's Society. Both of them had experienced recent marked cognitive decline, which worried Paula and June.

\section{Lessons Learned}

As other researchers have pointed out [19,20], recruitment strategy is of crucial importance. In our project, we worked with two large organizations who were extremely helpful in targeting potential participants and reviewing advertisements and documents necessary for ethical approval. These organisations were keen to involve their members in research but it became clear that timing of the recruitment was crucial so as not to interfere with other announcements, for example awareness month events, and overwhelm their members. This means very close coordination between support organizations and project team members, which needs to be planned in from the start.

We also decided to approach local dementia groups to speed up recruitment but local dementia groups were tricky to involve as they are often run by volunteers or casual staff, which makes contacting them difficult. Only about $10 \%$ of the support groups we approached responded. Once provided with more information, they were very happy for us to attend a support group meeting to present a formal talk about our project. This involved some additional, unplanned time and effort by one of the researchers. Other projects targeting vulnerable people should consider early on how to work closely with local support groups to establish early connections.

Recruitment should also take into account a representative sample. The advisory board to our project noted that often research is done with white, fairly affluent men and we were encouraged to consider how we could broaden involvement to other demographics. This is very difficult to achieve with small groups such as those in co-design, and we were only partially successful in attracting a diverse set of people. Recruiting people from a wider background would require 
more project resources and extending the recruitment period for much longer. We suggest that considerable effort is expended on this aspect of co-design.

\section{CO-DESIGN: EXPLORE, DESIGN AND EVALUATE}

The main part of our co-design consisted of four workshops, each lasting about three hours, spaced about six weeks apart over the course of six months, focusing on exploring, designing and evaluating steps:

- Workshop 1: exploring the background, technology use, activities and goals of users;

- Workshop 2: exploring the use of sensors and gaining input to the computational model;

- Workshop 3: designing the user interface using lowfidelity prototyping;

- Workshop 4: evaluating the user interface design using an adapted cognitive walkthrough.

Between workshops 1 and 2, participants used an empathy probe. After workshop 4, we conducted a round of user testing with co-design participants and others. In this persona-centred approach, persona construction and application were a common thread throughout all these workshops and steps.

\section{Exploring Users (Workshop 1 and Empathy Probes)}

In the first workshop, we were interested in exploring the background of the participants, including hobbies and interests, the technology that they interact with, and the activities and goals they do or would like to achieve. We also wanted to broaden the consideration of users beyond the codesign participants by using personas. A basic tenet of the PERCEPT approach is for target users to be directly involved in creating personas. To this end, we were inspired by the "someone who isn't me" (SWIM) technique [44], which has been employed with people with aphasia, a cognitive impairment that affects language understanding and production, and created a "stand-in" for people similar to themselves, from which the personas were derived. As part of our approach, we returned to these draft personas throughout workshop 1 to flesh them out, adapted them in workshop 2 and 3, and then used these personas to give structure to exercises and tasks. As initial facets for the personas we choose background, activities and goals, and technology, mirroring the important aspects for users of the

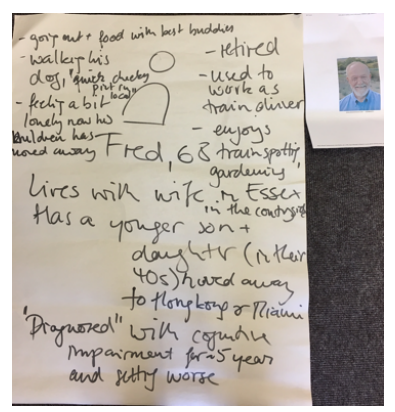

Figure 2. Fred, a draft persona for a person with dementia new system. We constructed these personas iteratively, always carrying out exercises with the participants to draw out their lived experiences focusing on these facets, and then we asked participants to flesh out the personas by reflecting and surfacing their experience.

First, participants and researchers introduced themselves to each other, talking about their background, interests and hobbies. The next exercise was to outline the personas, giving them a background. Participants collaborated to give these draft personas a name and background details, e.g. age, home location, family background and hobbies (Figure 2). A researcher was simply taking notes during this exercise; afterwards, the participants voted on images found online that might feasibly best represent the personas. In the first workshop, people with dementia and their informal carers developed three personas - Fred, his wife Vera and their daughter Enid. People living with Parkinson's disease created two personas, Steven and Pat.

We then focused on technologies, both for participants and the draft personas. First, the participants completed an individual activity to locate the technologies and devices that they interact with regularly in their homes and where they did so. They used a floorplan of a hypothetical small home that they could alter and annotate with technologies, either with pre-produced laminated cards of devices or by writing on the floorplan with a marker (Figure 3). At the end of this exercise, we asked what technologies and devices would be used by each of the draft personas.

Finally, we asked participants to brainstorm jointly about the goals and activities they want to achieve in their daily lives. Again, at the end of this exercise, we returned to the draft personas to flesh them out with goals and activities.

We asked participants to self-report on their daily activities, interests, challenges and difficulties they encountered between workshop 1 and 2 using empathy probes [15,21]. We provided a disposable film camera to each participant, and a pack for notes, although many participants decided to email digital photographs from their own devices. The photos and notes were used to enhance the personas in workshop 2 and to get a better idea of the participants lives outside of the co-design workshop.

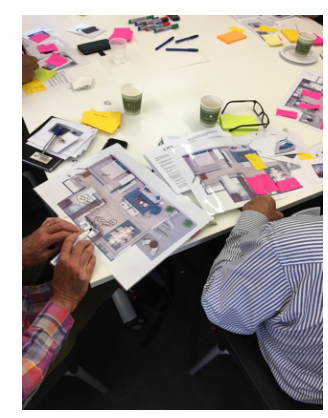

Figure 3. Co-design exercise using a floorplan of a small home to annotate it with technologies used at home 


\section{Lessons Learned}

We were pleasantly surprised how engaged participants were with developing the personas, given only minimal instructions. Their creation was characterized by playfulness and sometimes dark humour, while also being focused on the aspects relevant for technology design. For example, Prabhu suggested "Let's call him Shakin' Stevens" " from which one of the personas got its name, and it was also suggested that Steven's main interaction with technology is with a "beer fridge". In the dementia group, everybody had great fun putting together the background of the main persona's daughter, Enid, a wealthy socialite who swans about having lunches, while also providing details that she continues to care deeply about her father, phoning him every week to check on him. It also allowed participants to create a tangible artefact together very early on. What worked particularly well was breaking up the persona construction into smaller parts centring on the persona facets, and then interleaving the creation of personas with exercises that focused on the participants' own experiences.

One of the overriding consideration for any exercises was to minimize the need for handwriting, which can be challenging for people with Parkinson's and dementia. For example, we thought at length how to make the floorplan exercise accessible to both groups. We opted for large, laminated cards that showed common technology, to avoid reliance on fine motor control and to make them easier to handle. Assigning the writing to a researcher also encouraged the participants to externalize their thoughts more freely.

Overall, empathy probes produced mixed results. Some of the participants did not use them at all, saying that they did not feel that anything interesting was happening in their lives that merited taking photos. However, others relished showing us a snapshot how they lived. Figure 4 shows example images taken by participants, ranging from everyday encounters with technology that frustrated them, to pictures of their pets and loved ones, and places that they visited and were important to them. For co-design, the activities and technology documented through the probes were particularly interesting, especially as we could not

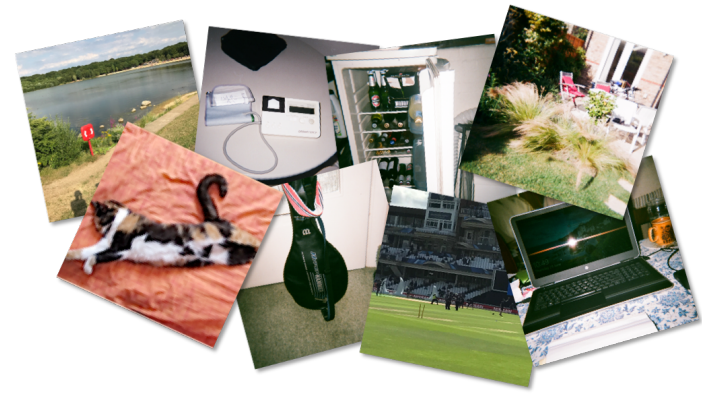

Figure 4. Example images taken by participants using empathy probes.

\footnotetext{
${ }^{2}$ A British rock and roll singer popular in the 1980 s.
}

include visits or sessions at their home, and they also fostered empathy with the people we were working with.

\section{Exploring Technology (Workshop 2)}

The second workshop had three parts: first, to review the data collected in workshop 1, and possibly to adapt the personas in light of this, second, to explore the evolving computational model of activities and goals, and its fit with participants' own experience, and third, to demonstrate how the sensors could be integrated into the technology and explore how users might employ them.

First, we reviewed the data collected in workshop 1 and the empathy probes, and provided an opportunity to reflect on and change the personas in light of this information. We first reminded participants about the personas they had created in workshop 1, and then a researcher went through an analysis of the data we had collected previously for the technology, and the activities and goals facets. After reviewing each facet, participants had an opportunity to change and update the personas.

For the technology facet of the persona, we analysed the floorplan annotations across all participants. We produced a word cloud of devices and technology for each group (Figure 5), and also a bar chart of a categorisation of the technologies and devices, e.g. refrigeration includes fridge, refrigerator and water cooler, cooking includes cooker, hob and microwave, etc. We then showed how this bar chart compared with the content in the personas to explore and stimulate reflection on differences and similarities (Figure 6). The participants in the Parkinson's group added a microwave and smart fridge for Pat, and a Bluetooth navigation system for Steven. Participants in the dementia group did not alter their personas because they felt that the personas matched them sufficiently.

For the activities and goals facet, we showed them their own data alongside the major categorizations in the evolving computational model (Figure 7). Participants in the Parkinson's group added shaving and dressing to Steven's activities to make him more like them, and sleeping and

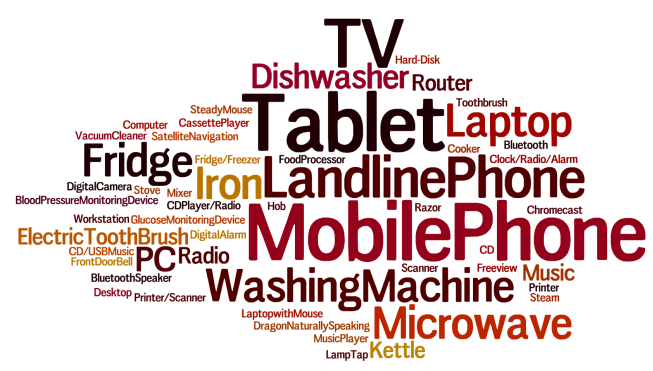

Figure 5. Example word cloud of floorplan annotations from the Parkinson's group. 


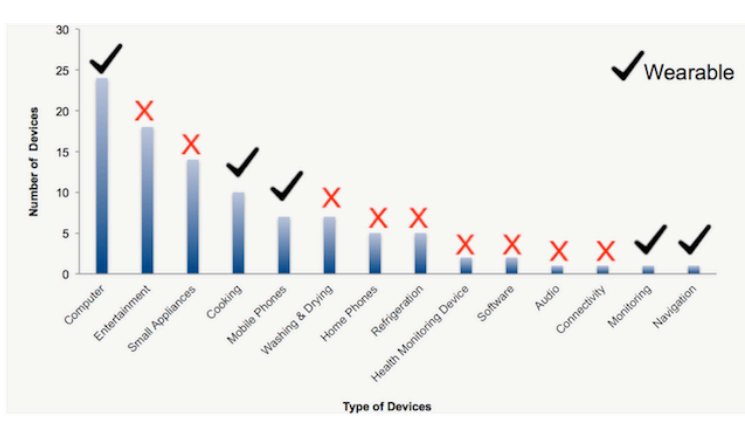

Figure 6. An example of the technology comparison between participants and the "Fred" persona for the dementia group. A $\checkmark$ denotes a match between participant data and the persona; $a \times$ means no match.

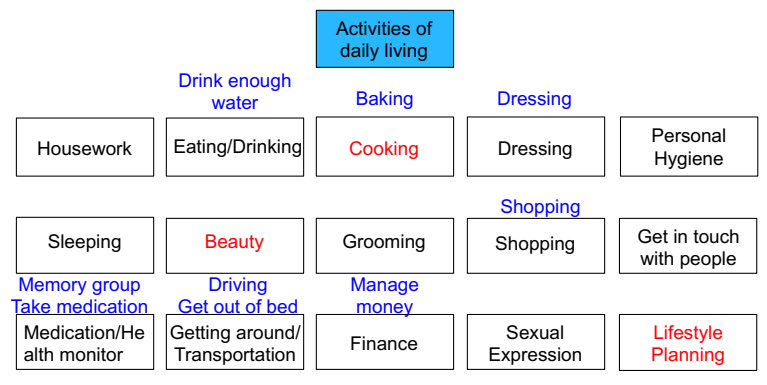

Figure 7. Activities of the dementia group participants mapped onto the evolving computational model. Blue means where this maps to an activity; red means a new activity was added based on participants' data.

grooming for professional appearance to Pat, while the dementia group was happy with their personas and they did not add any other activities to them.

In the second part, we conducted an exercise to explore the computational model's goals and activities and whether they made sense to the participants. To do this, we printed out all activities that we had collected from participants on paper. We also printed out the goals from the current computational model. The groups then brainstormed how their activities related to these goals, mapping complex relationships between them (Figure 8) and grouping the activities under the three main goals of the computational model. The participants also reviewed the terminology used in the naming of the goals. This information was then taken back into the design of the computational model.

The third part of workshop 2 explored how the sensor technology in our toolset could be used at home. A researcher first demonstrated what each sensor looked like, what it measured, and how it could be used to track different activities, using the group's main persona's activities as an example. In this exercise, participants tried to associate sensors that could be used to help them monitor their own activities. To structure this exercise, we prepared a "complete the blanks" sheet to capture objects they would like to track, the sensors they would use, the activities they might like to track and the goals that they would like to achieve (Figure 9). To fill in the blanks, they could place laminated cards or write in information that were not preprepared. Information from this exercise was used to add use cases for monitoring activities to the personas after the workshop.

\section{Lessons Learned}

The review of the data collected in workshop 1 and the comparison with the personas was useful to revise the personas. We kept the visualization of the analysis very simple which made understanding the information easy for participants. As a result of our experiences, we suggest that co-creation of personas include frequent staged reflection points to clarify with participants whether and how much the personas accord with current needs or future desires, continuously adapting and extending them as needed, based on quick and simple analysis. Involvement of a broader user base, perhaps through surveys, could also be useful to flesh out the personas created by users in a workshop.

The group exercise to engage participants with the computational model that was being developed alongside also proved fruitful. We were able to feed back design choices on the naming of goals and activities, and the model overall. For example, based on the workshop findings we

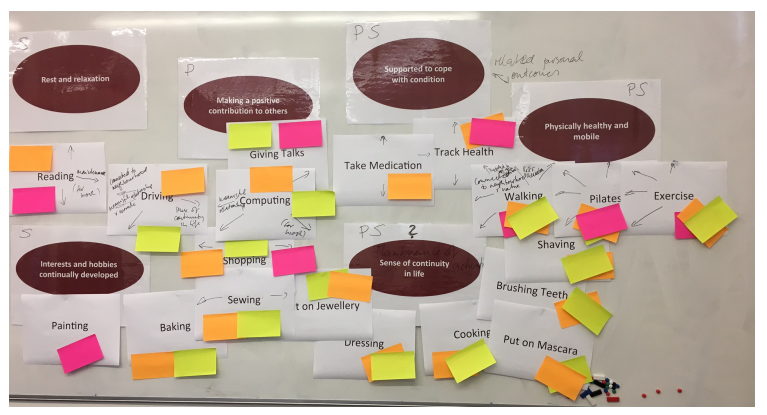

Figure 8. Participants mapped their activities to goals and reviewed the emerging computational model. Orange postits denote a main goal of "confidence and good morale in day to day life", yellow means "independence and choice and control over life", pink denotes "emotional and mental wellbeing".

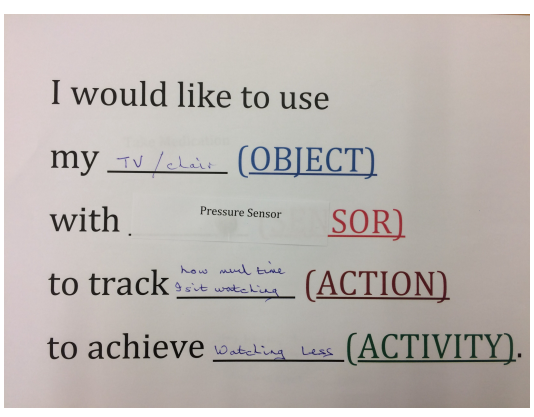

Figure 9. "Complete the blanks" exercise to draw out possible associations of sensors with activities. 
decided to reduce the complexity of the model exposed to users so as not to overwhelm them.

On the other hand, the exploration of the sensors was not as successful as it could have been. Participants struggled to understand how these sensors worked, and why they need to associate activities or actions with objects, instead hoping that "the system would do it for them". Possibly, we could have improved the co-design exercise providing a different format to draw out the potential use of these sensors, for example, using video prompts as in the OASIS approach [19] or using design probes [38].

\section{Designing a Low-fidelity Prototype (Workshop 3)}

Workshop 3 was dedicated to co-designing a user interface using low-fidelity prototyping with post-its, pens, different UI elements such as buttons, checkboxes, dropdown lists, text fields, date field, and blank A3 "screens". We used the previously developed personas' activities (e.g. shopping, going out, playing golf, painting, cooking, reading newspaper and exercising) and goals (e.g. maintain personal interests, maintain personal routines, achieve active mind, achieve relaxation, achieve everyday tasks and achieve physical activity) as a way to encourage participants to create the designs focusing on the wider user base, rather than drawing on their own personal preferences.

We focused on the main design concepts for setting up a life plan, monitoring progress against goals, and how to manage privacy or sharing of data. Participants with Parkinson's were mainly left to their own devices, starting from a blank page, while participants with dementia were more supported in designing, such as showing activities that the system could provide. In addition, a researcher drew what the participants in the dementia group were indicating, with the evolving design being critiqued by the group (Figure 10). The resulting low-fidelity prototypes were simple but indicated important functionality that the technology had to achieve, and metaphors that might be useful in the design.

\section{Lessons Learned}

The personas came into their own during paper-prototyping, and previous work has shown that personas created by researchers can be adopted successfully by co-design participants $[26,39,42]$. By making participants consider someone else, they were able to rise above their own personal

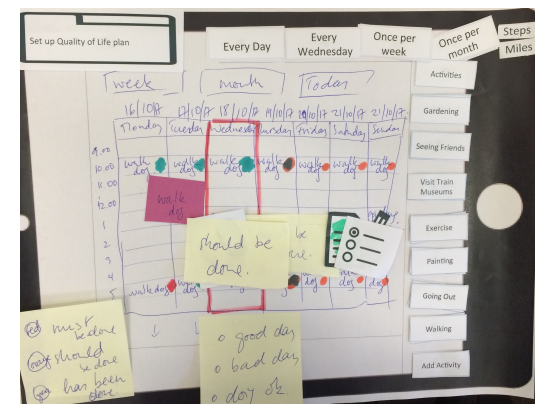

Figure 10. Calendar metaphor for setting up a life plan preferences and attitudes: "I don't see how EnableSelfCare could help me but I think it would definitely help Steven”, as Adam put it.

Low-fidelity prototyping is often used in co-design but there is not much research on how to adapt low-fidelity prototyping for user groups such as Parkinson's or dementia. Low-fidelity prototyping works best when it explores required functionality and metaphors that might be useful to adopt, instead of a blueprint for the interface. We tried to guide the design sensitively by suggesting how an interaction could be achieved but refraining from suggesting what functionality there should be. It is clear that there is a delicate balance to be struck along the spectrum between designers taking over from participants versus putting participants untrained in designing in charge [32,44].

Evaluating the Prototype (Workshop 4 and User Testing) We evaluated evolving prototypes in two ways: through an adapted cognitive walkthrough in workshop 4 and through user testing.

Our aim in workshop 4 was to evaluate a more refined prototypes that a researcher had created based on the paper prototypes and discussion in workshop 3. To do so, we were inspired by the GenderMag method [6] which combines the use of personas with a cognitive walkthrough to focus on gender differences in evaluating problem-solving software. In our approach, participants used the personas to step through a series of tasks and screens using an interactive prototype loaded onto a tablet computer. A researcher posed the following questions at each screen: "will $<$ Fred/Steven $>$ see what to do next", "will $<$ Fred/Steven $>$ realize that he $\mathrm{did}$ the right thing", and "will $<$ Fred/Steven $>$ know that he is making progress towards his goal?", following a simplified cognitive walkthrough method. As the participants discussed the interface in relation to the questions, a researcher took notes on an issue record form, adapted from the kit available at http://gendermag.org/.

After completing the co-design workshops, we integrated the feedback into the final designs and ran a series of user testing sessions, partly to check the interface designs are indeed usable by a wider sample of the population. Again, we integrated the use of the personas that we created during the co-design workshops. Ten participants, including eight people from the original co-design workshops, tested the interface designs on a tablet (Figure 11), focusing on different tasks such as set-up a profile, a life plan, sharing of their data with others, and how to monitor their life plan. The participants completed the user testing session in two parts: first to simulate use of the prototype by one of the co-created personas, and then to use it according to their own preferences. For the persona-based user testing, they were asked to perform tasks mimicking registering for the service, setting up a life plan, monitoring progress, and changing the life plan. Participants then set up activities relevant to them. The user testing session followed a typical think-aloud setup, followed by a debriefing interview, in which screen 


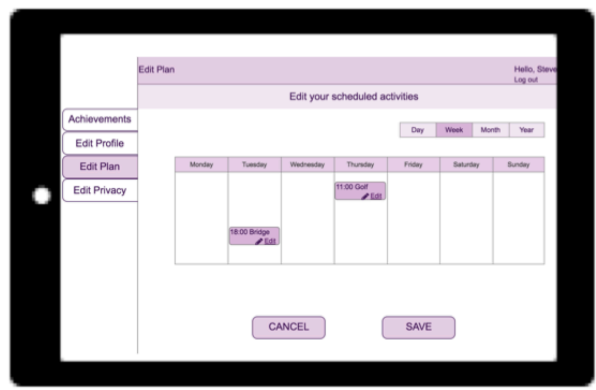

Figure 11. Participants tried out the final interface designs in user testing sessions.

activity, user expressions and audio were recorded and analysed for usability issues.

\section{Lessons Learned}

Based on our positive experiences, we suggest that personacentred evaluation in co-design is one of the strong points of the PERCEPT approach. Stepping systematically through an adapted cognitive walkthrough provided some structure for these participants, which became familiar quickly. By this time, the participants identified very strongly with the personas, and did not need to refer to the persona sheet even though it was available to them. In fact, participants with dementia who sometimes struggled to remember recent events indicated that they could remember everything about the personas.

To keep users engaged and envision the use of this technology in everyday life during user testing, we also supplemented the persona-driven tasks with their own individual context and tasks, so that the design would not be too specific to a certain persona and fail to highlight individual characteristics. We suggest that towards the end of co-designing, the use of personas might present a limitation and that broader user involvement is needed in developing and evaluating technology.

\section{THE PERSONAS}

We now present the personas that were developed and used by participants in our case study (Figure 12). We then analyse and discuss the strengths and weaknesses following the PERCEPT approach in Lessons Learned.

Participants in the Parkinson's group created two personas, "Steven" and "Pat", each representing different user groups of people with Parkinson's. Steven, the primary persona, is 64 years old, married and a retired fashion designer. 7 years ago, he was diagnosed with Parkinson's disease when he started having difficulties in buttoning up shirts at work. Steven is very social but he is having some difficulties in communicating with family and friends, and therefore he needs another mode of communication with them. He enjoys painting, BBQs with friends and family, playing golf and bridge, and going on holidays. Steven's goals are to keep being social and maintain his relationships with family and friends, but more immediately, to improve his golf playing and to feel less tired. Technology that Steven uses at home includes a smart phone with Bluetooth connectivity for his car, a tablet, a laptop, a desktop computer, a smart TV, video entry system and remote gates, and a SatNav system for his car. He also has a beer fridge. Sensor-based monitoring use cases were added to Steven after workshop 2; he would like to monitor when he gets up in the morning and therefore he uses a pressure and a motion sensor, he also uses a pedometer to track the number of steps he does when he plays golf.

Pat, the secondary persona with Parkinson's disease, is 53 years old, divorced, and works as a chief executive for a public health service. She was diagnosed with Parkinson's two years ago when she started having problems with the voice recognition system at work. Her most important goal is to continue with her career. Pat has a very busy lifestyle with no time for hobbies, but she really enjoys Pilates. She wants to manage her stress and balance her professional and personal life. Parkinson's makes her feel fatigued and she wants to manage her tiredness. Pat is very comfortable with technology and uses a smart phone, tablet, laptop, a smart watch for fitness and health, and a Global Positioning System (GPS). She also has access to a sophisticated home alarm system, smart metering system and smart fridge.

Participants living with dementia created three personas: Fred who has dementia, his wife Vera and their daughter Enid. The primary persona, Fred, has dementia and is a 68 year-old retired train driver. Fred has two grown-up children who live abroad. Five years ago, Fred was diagnosed with cognitive impairments and he has been getting worse since then. Fred is very social and he likes going out for dinner with his friends. Other hobbies are visiting train museums, walking his dog and gardening. His main goals are to maintain his relationships with family and friends, and keep being social. Fred uses a mobile phone with large separated keys and a large display with adjustable font size targeted to the senior market (e.g. a Doro) which his daughter bought him, a landline phone, a mobility scooter, a desktop computer, an alarm clock and a dog training whistle.

Vera, the secondary persona, is Fred's wife. She is 62 years old and works part-time at a local shop. Vera volunteers at a charitable organization and is involved in fundraising for dementia and cognitive impairments. She likes baking and aqua aerobics. Vera's main goal is to look after Fred and ensure that his daily routine is maintained, balancing his

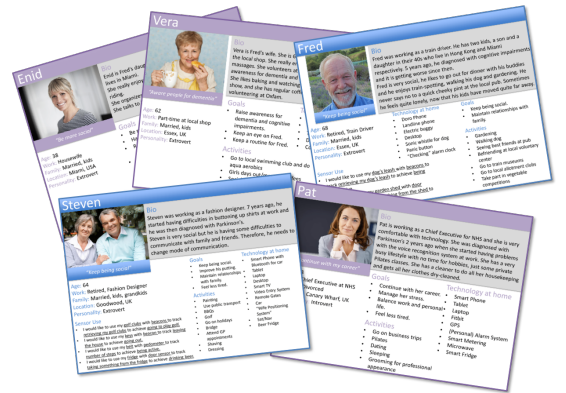

Figure 12. Personas created and used by participants in EnableSelfCare. 
short-term and long-term needs. Vera uses a mobile phone, a laptop, a TV and various cooking equipment including a digital scale.

The third persona is Enid, Fred and Vera's daughter. She is 38 years old, married and lives in Miami. Enid is very social and enjoys shopping and horse riding. She organizes galas to fundraise and increase awareness for Alzheimer's disease. Technology that Enid uses includes a smart phone, laptop, tablet, smart heating and lighting system, security and music systems.

At the start of workshop 3, participants with Parkinson's also added additional personas to simulate a wider informal care network, adapting the personas of Vera and Enid to their needs. Vera is now Steven's wife and raises awareness for Parkinson's disease. Enid, who is now Steven's daughter, organizes galas and fundraises for Parkinson's disease.

\section{Lessons Learned}

Recruitment needs to be carefully considered when creating personas. Participants in the Parkinson's workshop simply mirrored their group composition in the personas, without considering other stakeholders in the technology. We addressed this concern in workshop 3 but perhaps we could have outlined some additional stakeholder personas before the workshop. However, this would have run counter to our philosophy of putting the participants in charge.

A further limitation is that co-created personas might not explicitly reflect details that are obvious to the participants that created them or to people in similar situations i.e. "people-in-the-know". Participants did not include many signs and symptoms of dementia and Parkinson's disease, although it appears that there are subtle cues built into the personas themselves. Steven, for example, "has difficulty communicating" and Pat has issues with voice recognition; problems with speaking such as speech becoming slurred or unsteady being a symptom of Parkinson's. This lack of explicitness might become a problem if these personas are intended to be used outside of the co-creation group, or by designers and developers not familiar with the target user group and the chronic conditions.

On the other hand, there were also benefits to personas that are co-created by user groups themselves. Participants in the Parkinson's workshop extended the personas with unanticipated facets. For example, they pointed out that mental attitude towards living with the disease - what they termed "personality" - matters a great deal in attaining goals and sustaining activities.

\section{DISCUSSION AND CONCLUSION}

This paper introduced the PERCEPT (PERrsona-CEntred Participatory Technology) approach that employs co-created personas in exploring, designing and evaluating technology during co-design. Throughout, we answered the following research questions:
$R Q 1$ : How can we engage people with early stage dementia or Parkinson's, and their informal carers as equal research and design partners in co-design? $A$ : Participants enjoyed creating the personas, leading them to be deeply engaged with the personas they co-designed.

RQ2: Is it feasible to co-create personas with people with dementia and Parkinson's? A: Yes, but our paper has highlighted the limitations that researchers and practitioners need to consider when co-creating personas with target users, especially concerning representation of stakeholders and lack of explicitness.

$R Q 3:$ How can these personas be leveraged in the co-design process? A: Persona-centred co-design is especially useful in structuring the design and evaluation of prototypes and technologies. However, involvement and consideration of a broader user group is important as the technology is developed.

Our project has achieved a great deal in the space of 12 months using a co-design approach, trying to equalise the power between participants, researchers and designers. Of course, these timescales might be unrealistic in a commercial setting. We suggest that the process could be considerably speeded up by shortening the periods between co-design workshops; stages involving ethical considerations and recruitment could be shortened but adequate time should be ring-fenced for them.

This paper described a case study of co-designing the user interface of a self-care smart home technology toolset with people living with dementia or Parkinson's using the PERCEPT approach. We demonstrated in detail how we cocreated and used personas in this case study, and how we integrated them into techniques and exercises throughout codesign. We presented the personas that were created as part of our approach, and discussed their limitations. While our participants successfully employed the co-created personas during design and evaluation of the smart home toolkit, it is an open research question how much this approach can be leveraged by technology design projects dealing with other vulnerable groups. We encourage other researchers and designers for health and social care technologies to adopt participatory and co-design methods, and provide further feedback on the use of the PERCEPT approach. Our work presents a further step in developing methods and techniques for co-designing novel technologies, true to "nothing about us without us" [40].

\section{ACKNOWLEDGMENTS}

We thank our participants for their input to our project. The research reported in this paper is supported by the EPSRC Grant EP/P010024/1. We thank Julienne Meyer and the HCID research group for their constructive feedback.

\section{REFERENCES}

1. Tamara Adlin and John Pruitt. 2010. The Essential Persona Lifecycle: Your Guide to Building and Using Personas. Morgan Kaufmann. 
2. Amid Ayobi, Paul Marshall, Anna L. Cox, and Yunan Chen. 2017. Quantifying the Body and Caring for the Mind: Self-Tracking in Multiple Sclerosis. In Proceedings of the 2017 CHI Conference on Human Factors in Computing Systems (CHI '17), 6889-6901. https://doi.org/10.1145/3025453.3025869

3. Susanne Bødker and Ole Sejer Iversen. 2002. Staging a Professional Participatory Design Practice: Moving PD Beyond the Initial Fascination of User Involvement. In Proceedings of the Second Nordic Conference on Human-computer Interaction (NordiCHI '02), 11-18. https://doi.org/10.1145/572020.572023

4. Brian M. Bot, Christine Suver, Elias Chaibub Neto, Michael Kellen, Arno Klein, Christopher Bare, Megan Doerr, Abhishek Pratap, John Wilbanks, E. Ray Dorsey, Stephen H. Friend, and Andrew D. Trister. 2016. The mPower study, Parkinson disease mobile data collected using ResearchKit. Scientific Data 3. https://doi.org/10.1038/sdata.2016.11

5. DanaKai Bradford and Qing Zhang. 2016. How to Save a Life: Could Real-Time Sensor Data Have Saved Mrs Elle? In Proceedings of the 2016 CHI Conference Extended Abstracts on Human Factors in Computing Systems (CHI EA '16), 910-920. https://doi.org/10.1145/2851581.2851598

6. Margaret Burnett, Simone Stumpf, Jamie Macbeth, Stephann Makri, Laura Beckwith, Irwin Kwan, Anicia Peters, and William Jernigan. 2016. GenderMag: A Method for Evaluating Software's Gender Inclusiveness. Interacting with Computers 28, 6: 760787. https://doi.org/10.1093/iwc/iwv046

7. Jacob Buur and Susanne Bødker. 2000. From Usability Lab to "Design Collaboratorium": Reframing Usability Practice. In Proceedings of the 3rd Conference on Designing Interactive Systems: Processes, Practices, Methods, and Techniques (DIS '00), 297-307. https://doi.org/10.1145/347642.347768

8. Daniel G. Cabrero. 2014. Participatory Design of Persona Artefacts for User eXperience in non-WEIRD Cultures. In Proceedings of the 13th Participatory Design Conference: Short Papers, Industry Cases, Workshop Descriptions, Doctoral Consortium Papers, and Keynote Abstracts - Volume 2 (PDC '14), 247250. https://doi.org/10.1145/2662155.2662246

9. Daniel G. Cabrero, Heike Winschiers-Theophilus, Jose Abdelnour-Nocera, and Gereon Koch Kapuire. 2016. A Hermeneutic Inquiry into User-created Personas in Different Namibian Locales. In Proceedings of the 14th Participatory Design Conference: Full Papers Volume 1 (PDC'16), 101-110. https://doi.org/10.1145/2940299.2940310

10. Alan Cooper. 2004. The Inmates Are Running the Asylum: Why High Tech Products Drive Us Crazy and
How to Restore the Sanity (2Nd Edition). Pearson Higher Education.

11. J. Doyle, N. Caprani, and R. Bond. 2015. Older adults' attitudes to self-management of health and wellness through smart home data. In 2015 9th International Conference on Pervasive Computing Technologies for Healthcare (PervasiveHealth), 129-136. https://doi.org/10.4108/icst.pervasivehealth.2015.2592 79

12. Allison Druin. 1999. Cooperative Inquiry: Developing New Technologies for Children with Children. In Proceedings of the SIGCHI Conference on Human Factors in Computing Systems (CHI '99), 592-599. https://doi.org/10.1145/302979.303166

13. Shamal Faily and Ivan Flechais. 2011. Persona Cases: A Technique for Grounding Personas. In Proceedings of the SIGCHI Conference on Human Factors in Computing Systems (CHI '11), 2267-2270. https://doi.org/10.1145/1978942.1979274

14. Erin Friess. 2012. Personas and Decision Making in the Design Process: An Ethnographic Case Study. In Proceedings of the SIGCHI Conference on Human Factors in Computing Systems (CHI '12), 1209-1218. https://doi.org/10.1145/2207676.2208572

15. Bill Gaver, Tony Dunne, and Elena Pacenti. 1999. Design: Cultural Probes. interactions 6, 1: 21-29. https://doi.org/10.1145/291224.291235

16. Trisha Greenhalgh, Claire Jackson, Sara Shaw, and Tina Janamian. 2016. Achieving Research Impact Through Co-creation in Community-Based Health Services: Literature Review and Case Study. The Milbank Quarterly 94, 2: 392-429. https://doi.org/10.1111/1468-0009.12197

17. Aftab Khan, Sebastian Mellor, Eugen Berlin, Robin Thompson, Roisin McNaney, Patrick Olivier, and Thomas Plötz. 2015. Beyond Activity Recognition: Skill Assessment from Accelerometer Data. In Proceedings of the 2015 ACM International Joint Conference on Pervasive and Ubiquitous Computing (UbiComp '15), 1155-1166. https://doi.org/10.1145/2750858.2807534

18. Rashmi Lakshminarayana, Duolao Wang, David Burn, K. Ray Chaudhuri, Clare Galtrey, Natalie Valle Guzman, Bruce Hellman, Ben James, Suvankar Pal, Jon Stamford, Malcolm Steiger, R. W. Stott, James Teo, Roger A. Barker, Emma Wang, Bastiaan R. Bloem, Martijn van der Eijk, Lynn Rochester, and Adrian Williams. 2017. Using a smartphone-based self-management platform to support medication adherence and clinical consultation in Parkinson's disease. npj Parkinson's Disease 3, 1: 2. https://doi.org/10.1038/s41531-016-0003-z

19. Stephen Lindsay, Daniel Jackson, Guy Schofield, and Patrick Olivier. 2012. Engaging Older People Using 
Participatory Design. In Proceedings of the SIGCHI Conference on Human Factors in Computing Systems (CHI '12), 1199-1208.

https://doi.org/10.1145/2207676.2208570

20. Stephen Lindsay, Katie Brittain, Daniel Jackson, Cassim Ladha, Karim Ladha, and Patrick Olivier. 2012. Empathy, Participatory Design and People with Dementia. In Proceedings of the SIGCHI Conference on Human Factors in Computing Systems (CHI '12), 521-530. https://doi.org/10.1145/2207676.2207749

21. Tuuli Mattelmäki and Katja Battarbee. 2002. Empathy probes. In $P D C, 266-271$.

22. Tara Matthews, Tejinder Judge, and Steve Whittaker. 2012. How Do Designers and User Experience Professionals Actually Perceive and Use Personas? In Proceedings of the SIGCHI Conference on Human Factors in Computing Systems (CHI '12), 1219-1228. https://doi.org/10.1145/2207676.2208573

23. Jennifer (Jen) McGinn and Nalini Kotamraju. 2008. Data-driven Persona Development. In Proceedings of the SIGCHI Conference on Human Factors in Computing Systems (CHI '08), 1521-1524. https://doi.org/10.1145/1357054.1357292

24. Roisin McNaney, Madeline Balaam, Amey Holden, Guy Schofield, Daniel Jackson, Mary Webster, Brook Galna, Gillian Barry, Lynn Rochester, and Patrick Olivier. 2015. Designing for and with People with Parkinson's: A Focus on Exergaming. In Proceedings of the 33rd Annual ACM Conference on Human Factors in Computing Systems (CHI '15), 501-510. https://doi.org/10.1145/2702123.2702310

25. Kellie Morrissey, Andrew Garbett, Peter Wright, Patrick Olivier, Edward Ian Jenkins, and Katie Brittain. 2017. Care and Connect: Exploring DementiaFriendliness Through an Online Community Commissioning Platform. In Proceedings of the 2017 CHI Conference on Human Factors in Computing Systems (CHI '17), 2163-2174. https://doi.org/10.1145/3025453.3025732

26. Lene Nielsen. 2011. Personas in Co-creation and Codesign. In Proceedings of the 11th Danish HumanComputer Interaction Research Symposium (DHRS2011), 38-40.

27. Lene Nielsen and Kira Storgaard Hansen. 2014. Personas is Applicable: A Study on the Use of Personas in Denmark. In Proceedings of the SIGCHI Conference on Human Factors in Computing Systems (CHI '14), 1665-1674. https://doi.org/10.1145/2556288.2557080

28. Chimeren Peerbhai, Sylke Grootoonk, Hugo de Waal, Kate McLeish, and Derek L. Hill. 2016. Co-Designing a Digital Platform to Support People With Dementia And Their Carers. Alzheimer's \& Dementia 12, 7,
Supplement: P154-P155.

https://doi.org/10.1016/j.jalz.2016.06.254

29. John Pruitt and Jonathan Grudin. 2003. Personas: Practice and Theory. In Proceedings of the 2003 Conference on Designing for User Experiences (DUX '03), 1-15. https://doi.org/10.1145/997078.997089

30. Rebecca M. Quintana, Stephanie R. Haley, Adam Levick, Caitlin Holman, Ben Hayward, and Mike Wojan. 2017. The Persona Party: Using Personas to Design for Learning at Scale. In Proceedings of the 2016 CHI Conference Extended Abstracts on Human Factors in Computing Systems (CHI EA '17), 933-941. https://doi.org/10.1145/3027063.3053355

31. Louise Robinson, Katie Brittain, Stephen Lindsay, Dan Jackson, and Patrick Olivier. 2009. Keeping In Touch Everyday (KITE) project: developing assistive technologies with people with dementia and their carers to promote independence. International Psychogeriatrics 21, 3: 494-502. https://doi.org/10.1017/S1041610209008448

32. Elizabeth B.-N. Sanders and Pieter Jan Stappers. 2008. Co-creation and the new landscapes of design. CoDesign 4, 1: 5-18. https://doi.org/10.1080/15710880701875068

33. Michael Scaife, Yvonne Rogers, Frances Aldrich, and Matt Davies. 1997. Designing for or Designing with? Informant Design for Interactive Learning Environments. In Proceedings of the ACM SIGCHI Conference on Human Factors in Computing Systems (CHI '97), 343-350. https://doi.org/10.1145/258549.258789

34. Helena Sustar, Simon Bowen, Andy Dearden, Mark Fisher, and Dan Wolstenholme. 2013. Using popular culture to enable health service co-design with young people. In Crafting the Future.

35. Anja Thieme, John Vines, Jayne Wallace, Rachel Elizabeth Clarke, Petr Slovák, John McCarthy, Michael Massimi, and Andrea Grimes Grimes Parker. 2014. Enabling Empathy in Health and Care: Design Methods and Challenges. In CHI '14 Extended Abstracts on Human Factors in Computing Systems (CHI EA '14), 139-142. https://doi.org/10.1145/2559206.2559237

36. John Vines, Roisin McNaney, Rachel Clarke, Stephen Lindsay, John McCarthy, Steve Howard, Mario Romero, and Jayne Wallace. 2013. Designing For- and With- Vulnerable People. In CHI '13 Extended Abstracts on Human Factors in Computing Systems (CHI EA '13), 3231-3234. https://doi.org/10.1145/2468356.2479654

37. Jayne Wallace, Anja Thieme, Gavin Wood, Guy Schofield, and Patrick Olivier. 2012. Enabling Self, Intimacy and a Sense of Home in Dementia: An Enquiry into Design in a Hospital Setting. In 
Proceedings of the SIGCHI Conference on Human Factors in Computing Systems (CHI '12), 2629-2638. https://doi.org/10.1145/2207676.2208654

38. Jayne Wallace, Peter C. Wright, John McCarthy, David Philip Green, James Thomas, and Patrick Olivier. 2013. A Design-led Inquiry into Personhood in Dementia. In Proceedings of the SIGCHI Conference on Human Factors in Computing Systems (CHI '13), 2617-2626. https://doi.org/10.1145/2470654.2481363

39. Pontus Wärnestål, Petra Svedberg, Susanne Lindberg, and Jens M Nygren. 2017. Effects of Using Child Personas in the Development of a Digital Peer Support Service for Childhood Cancer Survivors. Journal of Medical Internet Research 19, 5. https://doi.org/10.2196/jmir.7175

40. David Werner. 1998. Nothing About Us Without Us: Developing Innovative Technologies For, By, and With Disabled Persons. Healthwrights, Palo Alto.

41. Peter West, Richard Giordano, Max Van Kleek, and Nigel Shadbolt. 2016. The Quantified Patient in the
Doctor's Office: Challenges \& Opportunities. In Proceedings of the 2016 CHI Conference on Human Factors in Computing Systems (CHI '16), 3066-3078. https://doi.org/10.1145/2858036.2858445

42. Irith Williams, Margot Brereton, Jared Donovan, Karalyn McDonald, Tanya Millard, Alex Tam, and Julian H. Elliott. 2014. A collaborative rapid personabuilding workshop : creating design personas with health researchers. International Journal of Sociotechnology and Knowledge Development 6, 2: 17-35.

43. Stephanie Wilson, Abi Roper, Jane Marshall, Julia Galliers, Niamh Devane, Tracey Booth, and Celia Woolf. 2015. Codesign for people with aphasia through tangible design languages. CoDesign 11, 1: 21-34. https://doi.org/10.1080/15710882.2014.997744

44. Peter Wright and John McCarthy. 2008. Empathy and Experience in HCI. In Proceedings of the SIGCHI Conference on Human Factors in Computing Systems (CHI '08), 637-646. https://doi.org/10.1145/1357054.1357156 